Gynecologic and

Obstetric Investigation
Gynecol Obstet Invest 2012;74:304-312

DOI: $\underline{10.1159 / 000339632}$
Received: November 17, 2011

Accepted after revision: May 22, 2012

Published online: August 10, 2012

\title{
A Linear Karyotypic Association between PB-I, PB-II and Blastomere Using Sequentially Performed Comparative Genome Hybridization with No Association Established between Karyotype, Morphologic, Biochemical (sHLA-G Expression) Characteristics, Blastocyst Formation and Subsequent Pregnancy Outcome
}

\author{
Dirk Kotze $^{a} \quad$ Levent Keskintepe $^{a} \quad$ Geoffrey Sher ${ }^{a}$ Thinus Kruger ${ }^{b}$ \\ Carl Lombard ${ }^{c}$

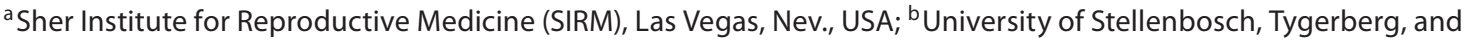 \\ 'Medical Research Council, Cape Town, South Africa
}

\section{Key Words}

Polar body - Blastomere - Whole genome amplification •

Comparative genome hybridization • Aneuploidy

\begin{abstract}
Background: The importance of oocyte/embryo ploidy to achieve implantation and a subsequent pregnancy. Aim: To correlate first and second polar bodies and day-3 blastomere ploidy, embryo morphology and biochemical (SHLA-G) characteristics with blastocyst development and in vitro pregnancy outcome. Materials and Methods: All oocytes/zygotes and embryos were individually cultured to the blastocyst stage. PB-I, PB-II and blastomeres underwent complete karyotyping and sHLA-G expression was measured on day 2. Results: 57 mature (MII) donor oocytes were obtained, 33/57 (57.9\%) were aneuploid, 21/57 (36.8\%) were euploid, and $3 / 57$ (5\%) were 'inconclusive'. No correlation was found between comparative genomic hybridization (CGH) status of PB-I, PB-II and the graduated embryo score. Furthermore, no correlation was established between PB-I CGH results and blastocyst morphology grade. There was a significant cor-
\end{abstract}

\section{KARGER}

Fax +4161306 1234

E-Mail karger@karger.ch

www.karger.com
(C) 2012 S. Karger AG, Basel

$0378-7346 / 12 / 0744-0304 \$ 38.00 / 0$

Accessible online at:

www.karger.com/goi relation between PB-I CGH and blastomere CGH results. Euploid and aneuploid PB-I developed into 58 and $67 \%$ blastocysts, respectively. $\kappa$ statistics $(>0.7)$ revealed a positive correlation between the ploidy of PB-I, PB-II and the blastomeres. Conclusion: Following ICSI and sequential genetic karyotyping of the oocyte/zygote and subsequent blastomeres, the majority of oocytes fertilized and subsequent zygotes developed into blastocysts, despite their ploidy status. We therefore conclude that blastocyst development is not associated with ploidy.

Copyright $\odot 2012$ S. Karger AG, Basel

\section{Introduction}

Several factors are involved in achieving successful embryo implantation and a subsequent pregnancy. Poor embryo quality [1] and transfer techniques [2], endouterine abnormalities and immunologic dysfunction [3] can lead to implantation failure. Noninvasive methods such as embryo morphology/scoring and extended embryo culture to the blastocyst stage have been proposed to se- 
lect embryos for transfer that will implant and result in a live pregnancy. Furthermore, a biochemical marker - soluble human leukocyte-antigen-G expression (sHLAG) - major histocompatibility complex - by embryos in culture has been suggested by several researchers to improve assisted reproductive technology outcome.

However, all the above-mentioned criteria provide no information about the chromosomal status (ploidy) of an embryo, which is of utmost importance because certain chromosomal aberrations in the embryo might contribute to in vitro fertilization (IVF) failure [4]. Early studies using preimplantation genetic screening with fluorescent in situ hybridization (PGS-FISH) have demonstrated that $\geq 50 \%$ of embryos considered for transfer during IVF were aneuploid [5]. In the past, FISH has been the most common method used for analyzing polar body (PB) and blastomere chromosomal complements. Initially it was suggested that the transfer of 'chromosomally normal' embryos after PGS-FISH would lead to improved implantation and pregnancy rates. However, recent publications [6] (ESHRE PGD Consortium Steering Committee, 2010) have concluded otherwise. One of the shortfalls associated with FISH in a PGS setting is the limited number of chromosomes that can be analyzed simultaneously; this is due to the number of fluorochromes available. Therefore, since FISH only screens up to 12 chromosomes, it limits the screening of the entire human genome, consisting of 23 chromosome pairs of which 22 chromosomes are autosomal chromosomes and the remaining pair is sexdetermining $(\mathrm{X}, \mathrm{Y})$. Other potential problems like mosaicism is still an issue when performing day-3 biopsies (although mosaicism has been detected at later developmental stages), as well as the removal of more than one blastomere, leaving less of the remaining embryo. Studies have been reported where PGS-FISH was performed on polar bodies using a limited number of probes $[7,8]$. Comparative genomic hybridization (CGH) is another method for identifying oocyte/zygote/embryo chromosomal aberrations. The first studies using PB-CGH [9-11] failed to demonstrate any promise of practical clinical utility. It was not until Sher et al. [12] reported the first clinical application of CGH performed on both PB-I and PB-II. The same research group reported on the positive correlation between the transfer of euploid embryos (based on CGH performed on day-3 blastomeres) and IVF outcome [13]. The advantage of $\mathrm{CGH}$ is that the entire chromosomal complement/genome is analyzed. However, a downside is that polyploidy and balanced translocations cannot be detected reliably using metaphase CGH as described by other groups $[14,15]$. Furthermore, an initial disadvantage of
CGH was that the procedure took about $72 \mathrm{~h}$ to perform. Originally it was applied to polar bodies, leaving 5 days for analysis and allowing for a fresh embryo transfer, without having to cryopreserve the embryos prior to transfer. When blastomere biopsies were performed on day-3 embryos (6-9 cells), the resulting blastocysts were individually cryopreserved/vitrified and transferred in a later cycle if identified as normal by Sher et al. [13].

The aim of this study is to correlate PB-I, PB-II and day-3 blastomere ploidy, morphologic and biochemical (sHLA-G expression) characteristics with blastocyst development in vitro and pregnancy outcome.

\section{Materials and Methods}

\section{Patients}

Oocyte donors for this study were 5 healthy, young ( $\leq 28$ years of age), consenting patients without reproductive problems. Sperm from proven donors (with a record of successfully fathering a pregnancy) were obtained from Cryogam bank (Cryogam, Loveland, Colo., USA). All donors were appropriately screened for infectious and sexually transmitted diseases and for autoimmune parameters that might adversely affect embryo implantation.

\section{Embryo Culture}

Embryology data collection: All oocytes collected were kept in chronological sequence and were individually cultured in $100 \mu \mathrm{l}$ modified human tubal fluid (mHTF) $+10 \%$ HSA, under oil at $37^{\circ} \mathrm{C}$. All oocytes were denuded immediately after retrieval and graded [16] (table 1). PB-I was microsurgically removed and underwent whole genome amplification (WGA), followed by complete karyotyping using metaphase CGH. All mature oocytes (MII) were fertilized by intracytoplasmic sperm injection (ICSI) and sequentially cultured in $50-\mu$ l droplets of Quinn's Advantage Protein Plus Cleavage medium (In-Vitro Fertilization, Inc., Trumbull, Ct., USA) under oil at $6 \% \mathrm{CO}_{2}, 5 \% \mathrm{O}_{2}$ and $90 \% \mathrm{~N}_{2}$, $37^{\circ} \mathrm{C}$ in a humidified environment. On day 1 , fertilization was assessed $16-18 \mathrm{~h}$ post-ICSI, at which time alignment of the nucleoli was documented, and subsequently signs of cleavage at $26 \mathrm{~h}$ post-ICSI. At this time, PB-II was microsurgically removed from normally fertilized (2PN) oocytes and underwent WGA/CGH karyotyping. On day 2 (at $46 \mathrm{~h}$ post-ICSI) the embryos were moved into 50- $\mu \mathrm{l}$ droplets of Multi Blastocyst medium (Global one, IVF Online) + 10\% SSS (Irvine Scientific, Santa Ana, Calif., USA). The original droplets of culture medium surrounding each individual embryo were collected and tested for sHLA-G expression, using a specific enzyme-linked immunosorbent assay (ELISA). Embryos with an optical density range of $0.190 \pm 0.006$ were considered 'positive' for the expression of sHLA-G. Each individually cultured embryo was evaluated at $72 \mathrm{~h}$ post-ICSI - applying a graduated embryo score (GES) described by Fisch et al. [17] (table 2). Then, a single mononucleated blastomere was microsurgically removed from each cleaved embryo and underwent WGA/ $\mathrm{CGH}$ karyotyping. All embryos were cultured to the blastocyst stage and graded, using a modified version of the procedure [18] (table 3), and vitrified. 
Table 1. Oocyte grading used for the CGH study

Grade 1: homogenous cytoplasm, intact PB, normal oocyte shape, no cytoplasmic defect, no PV and zonae defect, normal oolemma

\section{following: \\ Fragmented PB \\ Abnormal oocyte shape \\ Cytoplasmic droplets \\ Vacuoles \\ Grainy spots \\ Increased PV space \\ Darken zonae or defect \\ Double oolemma}

Grade 2: homogenous cytoplasm, and at least two of the

Grade 3: no homogenous cytoplasm, and at least 3 of the above

Table 2. GES of cleavage stage embryos [from 17]

\begin{tabular}{|c|c|c|c|}
\hline Evaluation & $\begin{array}{l}\text { Hours after } \\
\text { insemination }\end{array}$ & Developmental milestone & Score \\
\hline 1 & $16-18$ & $\begin{array}{l}\text { Nucleoli aligned along } \\
\text { pronuclear axis }\end{array}$ & 20 \\
\hline \multirow[t]{4}{*}{2} & $25-27$ & $\begin{array}{l}\text { Cleavage regular and } \\
\text { symmetrical fragmentation }^{\mathrm{a}}\end{array}$ & 30 \\
\hline & & Absent & 30 \\
\hline & & $<20 \%$ & 25 \\
\hline & & $>20 \%$ & 0 \\
\hline \multirow[t]{2}{*}{3} & $64-67$ & $\begin{array}{l}\text { Cell number and grade }{ }^{\mathrm{b}} 7 \mathrm{CI} \text {, } \\
8 \mathrm{CI}, 8 \mathrm{CII}, 9 \mathrm{CI}\end{array}$ & 20 \\
\hline & & $\begin{array}{l}\text { 7CII, 9CII, 10CI, 11CI, } \\
\text { Compacting I }\end{array}$ & 10 \\
\hline
\end{tabular}

Total score

100

a If the embryo was not cleaved at 25-27 h, grading of fragmentation should occur at the 64-67-hour evaluation if the embryo reached the 7 -cell stage and had $<20 \%$ fragmentation.

${ }^{\mathrm{b}}$ Grade I = Symmetrical blastomeres and absent fragmentation; grade II = slightly uneven blastomeres and $<20 \%$ fragmentation; grade III = uneven blastomeres and $>20 \%$ fragmentation; grade A embryos are 7 or more cells with $<20 \%$ fragmentation.

\section{Blastocyst Vitrification and Warming}

Vitrification was done by modifying the method previously described by Mukaida et al. [19]. This modified technique involved the use of an ICSI needle as follows: About $10 \mathrm{~min}$ prior to vitrification, expanded blastocysts were placed in 50- $\mu$ l drops of $\mathrm{mHTF}$ with $10 \% \mathrm{SSS}$, vol/vol (Irvine Scientific). Each blastocyst had its blastocoels artificially collapsed through assertive needle aspiration. The inner cell mass was positioned at the 6- or
Table 3. Blastocyst grading

Blastocyst grading according to Dokras et al. [18] was performed as follows:

Grade 1 blastocysts were characterized by early cavitation resulting in the formation of an eccentric and then expanded cavity lined by a distinct inner cell mass region and trophectoderm layer

Grade 2 blastocyst exhibited a transitional phase where single or multiple vacuoles were seen which over subsequent days developed into the typical blastocyst appearance of a grade 1 blastocyst

Grade 3 blastocysts were defined as blastocysts with several degenerative foci in the inner cell mass with cells appearing dark and necrotic

12-o'clock position using a holding pipette connected to a micromanipulator. A $30^{\circ}$ ICSI needle was then introduced via the cellular junction of the trophectoderm into the blastocoel cavity and the fluid was aspirated until the blastocyst had completely collapsed and no blastocoel fluid remained $[19,20]$. Blastocysts were then placed in $0.5 \mathrm{ml}$ of $\mathrm{mHTF}$ (base solution; IVF Online) supplemented with $10 \%$ (vol/vol) SSS for $2 \mathrm{~min}$ at $37^{\circ} \mathrm{C}$. Thereafter, blastocysts were placed in base solution $+0.96 \mathrm{~mol} / \mathrm{l} \mathrm{DMSO}$ (D 5879; Sigma, St. Louis, Mo., USA) + $1.2 \mathrm{~mol} / \mathrm{l}$ ethylene glycol (P 3265; Sigma) for 90 s. Finally, blastocysts were placed in base solution + $1.9 \mathrm{~mol} / \mathrm{l} \mathrm{DMSO}+2.4 \mathrm{~mol} / \mathrm{l}$ ethylene glycol $+1 \mathrm{~mol} / \mathrm{l}$ sucrose (S 7903; Sigma) + $0.1 \mathrm{~mol} / 1$ Ficoll (F 8636; Sigma) for $30 \mathrm{~s}$. Then each blastocyst was individually placed onto a cryoloop (Hampton Research, Calif., USA) using 1-2 $\mu$ l cryosolution and plunged into liquid nitrogen. For the blastocyst grading applied, see table 3.

Warming. The cryoloop was removed from its vial and immersed into base solution $+0.34 \mathrm{~mol} / 1$ sucrose for $2 \mathrm{~min}$ at $37^{\circ} \mathrm{C}$. The embryo was then moved to base solution $+0.17 \mathrm{~mol} / \mathrm{l}$ sucrose for $3 \mathrm{~min}$ and finally placed in base solution for $5 \mathrm{~min}$. Intact embryos were cultured in $50 \mu \mathrm{l}$ of Global One medium at $37^{\circ} \mathrm{C}$ in $6 \%$ $\mathrm{CO}_{2}$ for a minimum of $2 \mathrm{~h}$ to evaluate blastocoel re-expansion (i.e. survival).

\section{Recipient Hormonal Treatment and Embryo Transfer}

Following the onset of birth control pill-induced menstruation, estradiol valerate IM (4-8 mg) was administered every 3 days for a period of 8-12 days, until endometrial thickness had reached $>8 \mathrm{~mm}$ in sagittal diameter and the plasma $\left(\mathrm{E}_{2}\right)$ had stabilized at 1,300-3,000 pmol/l. At that point, daily IM injections of $100 \mathrm{mg}$ progesterone in oil were initiated. On the 6th day of progesterone in oil, subject to patient choice and availability, two thawed/warmed blastocysts were cryotransferred, using ultrasound guidance and an echogenic catheter (Wallace, Smith Medical, UK). Serum $\beta$-human chorionic gonadotropin ( $\beta$-hCG) levels were measured 8-10 days after the transfer. The patient was considered biochemically pregnant when the first value was $>5.0$ IU and the next value 2 days later was double the first. Clinical 
pregnancy rates were based on a 6-week ultrasound scan detecting a gestational sac containing a fetal heartbeat. In the event of an ultrasound-confirmed pregnancy, an estrogen/progesterone supplementation regimen was continued. Ongoing pregnancy rates were based on a 10- to 12 -week ultrasound examination. In all cases where pregnancy did not occur or did not survive, hormonal treatment was immediately stopped.

Each blastocyst was referenced back to its oocyte, zygote and embryo of origin. All clinical procedures were performed by the same physician and laboratory procedures were performed by the same embryologist.

\section{Stimulation}

Patients received Lupron (TAP Pharmaceuticals, Lake Forest, Ill., USA) in a long protocol after pretreatment with oral contraceptive birth control pills for 1-3 weeks and were treated with a human-derived gonadotropin (Bravelle; Ferring Pharmaceuticals Inc., Suffern, N.Y., USA) in order to stimulate follicular development in the ovaries. Ovulation was triggered when at least two follicles were $18 \mathrm{~mm}$ and half of the remaining were $15 \mathrm{~mm}$. Oocytes were retrieved transvaginally using ultrasound guidance at $35 \mathrm{~h}$ post-human chorionic gonadotropin.

\section{Extraction and Amplification of Genomic DNA}

Genomic DNA was obtained by laser dissection and needle aspiration from three sources: the oocyte's first PB (PB-I), zygote's second $\mathrm{PB}$ (PB-II), and subsequent day-3 embryo's blastomere. The cellular material was aspirated into a 200 - $\mu$ l thin-walled PCR tube (VWR catalog, 82006-602) for lyses and amplification using the Qiagen Repli-g kit (catalog, 59045). 10 ng of Repli-g control genomic DNA was used as a positive control and $0.5 \mu \mathrm{l}$ of nuclease-free water as a negative control (Eppendorf catalog, 955155017). The reactions were set up following the Repli-g protocol and incubated for $8 \mathrm{~h}$ at $30^{\circ} \mathrm{C}$ for amplification, followed by $10 \mathrm{~min}$ at $70^{\circ} \mathrm{C}$ for enzyme inactivation. Both incubation steps were performed using an Applied Biosystems 2720 thermal cycler. Amplified DNA was placed at either $4^{\circ} \mathrm{C}$ for short-term storage or at $-20^{\circ} \mathrm{C}$ for long-term storage.

\section{Genomic DNA Confirmation and Quantification}

For confirmation of WGA products, $5-\mu$ l reaction aliquots were mixed with $1 \mu \mathrm{l} 6 \times$ Blue-Orange Loading Dye (Promega \# G1881) and electrophoresed through a $1.0 \%$ agarose gel containing $0.1 \%$ ethidium bromide in TBE buffer $(90 \mathrm{mM}$ Tris- $\mathrm{HCl}, 90$ $\mathrm{mM}$ boric acid and 2 mM EDTA, $\mathrm{pH}$ 8.0). For quantification of the genomic DNA, 5- $\mu$ l reaction aliquots were diluted with $95 \mu \mathrm{l}$ of molecular biology-grade, nuclease-free water and analyzed with an Eppendorf BioPhotometer.

\section{Nick Translation of Genomic DNA}

The WGA products were fluorescently labeled using the Vysis Nick Translation Kit (catalog, 32-801024). $1 \mu$ g genomic DNA for each sample was labeled with SpectrumGreen ${ }^{\mathrm{TM}}$ direct-labeled dUTP by nick translation, following the Vysis protocol. Translation reactions were carried out using an Applied Biosystems 2720 thermal cycler at $15^{\circ} \mathrm{C}$ for $2 \mathrm{~h}$ and $70^{\circ} \mathrm{C}$ for $10 \mathrm{~min}$ to stop the reaction. The size of the resulting SpectrumGreen ${ }^{\mathrm{TM}}$-labeled probes was assessed by electrophoresis of $5-\mu l$ aliquots of $1.0 \%$ agarose gel containing $0.1 \%$ ethidium bromide in TBE buffer $(90 \mathrm{mM}$ Tris$\mathrm{HCl}, 90 \mathrm{~mm}$ boric acid and $2 \mathrm{~mm}$ EDTA, pH 8.0). Probes of usable quality were in the range of $300-3,000 \mathrm{bp}$, as per the manufacturer's recommendation.

\section{Probe Preparation and Comparative Genomic Hybridization}

The SpectrumGreen ${ }^{\mathrm{TM}}$-labeled probes were purified by ethanol precipitation. Briefly, $10 \mu \mathrm{l} / 200 \mathrm{ng}$ nick-translated reference DNA, $1 \mu \mathrm{l} / 100$ ng SpectrumRed ${ }^{\mathrm{TM}}$ reference DNA (Vysis \# 3280423 or $32-804024$ ), and $10 \mu \mathrm{l} / 10 \mu \mathrm{g}$ Cot-1 DNA (Vysis \# 32800028 ) used to suppress repetitive sequences and prevent nonspecific hybridization, were added to a $1.5-\mathrm{ml}$ microcentrifuge tube.

For precipitation of the hybridization mix, $2.1 \mu \mathrm{l}(0.1 \mathrm{vol}) 3 \mathrm{M}$ sodium acetate, followed by $52.5 \mu \mathrm{l}(2.5 \mathrm{vol})$ of $100 \% \mathrm{EtOH}$ was added to the DNA, vortexed briefly and placed on dry ice for 15 min, then centrifuged at $12,000 \mathrm{rpm}$ for $30 \mathrm{~min}$ at $4^{\circ} \mathrm{C}$ to pellet the DNA. The supernatant was removed and the pellet dried for 10-15 min under a vacuum at ambient temperature. The pellet was resuspended in $3 \mu \mathrm{l}$ molecular biology-grade water and $7 \mu \mathrm{l}$ CGH hybridization buffer (Vysis \# 30670003) and placed in the dark during slide preparation. CGH-normal metaphase slides (Vysis \# 30-806010) were prepared following the manufacturer's protocol. Briefly, the slides were denatured in $70 \%$ formamide, $10 \%$ SSC pH 5.3, and 20\% molecular biology-grade water for $5 \mathrm{~min}$ at $73^{\circ} \mathrm{C}$ for $5 \mathrm{~min}$ followed by an ethanol dehydration series of a minute each $(70,85$, and $100 \%)$. The slides were dried by touching the bottom edge to a blotter and wiping the underside with a paper towel, then placed on a $45-50{ }^{\circ} \mathrm{C}$ slide warmer to allow remaining EtOH to evaporate. The probe mix was denatured by heating for $5 \mathrm{~min}$ in a $73^{\circ} \mathrm{C}$ water bath. $10 \mu \mathrm{l}$ of the probe mix was applied to the slides. The coverslip was placed over the slide and sealed with diluted rubber cement. The hybridizations were carried out with Vysis Hybrite chambers for a minimum of $48 \mathrm{~h}$ and a maximum hybridization time of $72 \mathrm{~h}$ to clear background. Following hybridization, the coverslips were carefully removed along with any remaining rubber cement residue, taking care not to damage the slide surface. The slides were then agitated in $0.4 \times$ SSC $/ 0.3 \% \mathrm{NP}-40$ wash solution at $74+1^{\circ} \mathrm{C}$ for $1-3 \mathrm{~s}$, then let stand for $2 \mathrm{~min}$, then agitated in $2 \times \mathrm{SSC} / 0.1 \% \mathrm{NP}-40$ at ambient temperature for $1-3 \mathrm{~s}$, then let stand for $1 \mathrm{~min}$. The slides were allowed to dry vertically at ambient temperature in the dark. Once completely dry, $10 \mu$ l DAPI II counterstain (Vysis 30804931) was added to each side, followed by a coverslip, and immediately sealed with clear nonfluorescing fingernail polish [21], collected and validated results from first, second PBs and two blastomeres and reported a positive/negative occurrence of less that $10 \%$.

\section{Image Capture and Analysis}

The following optical filters visualized the fluorochromes used in the hybridization: a filter set specific for DAPI, Texas Red ${ }^{\circledR}$ (Cat. No. 30-150491) and FITC (fluorescein isothiocyanate) (Cat. No. 30-150291) to view the counterstain, SpectrumRed ${ }^{\mathrm{TM}}$ or SpectrumGreen ${ }^{\mathrm{TM}} \mathrm{DNA}$, respectively, a triple band pass filter set designed to simultaneously excite and emit light specific for DAPI.

\section{Soluble HLA-G Assay}

A monoclonal antibody (mAb; MEM-G9 MCA 2044; Serotec, Raleigh, N.C., USA) against sHLA-G was used to coat a 96-well Nunc-Immunoplate (Fisher Scientific, Chino, Calif., USA) using a concentration of $2 \mu \mathrm{g} / \mathrm{ml}$ in $0.1 \mathrm{~mol} / \mathrm{l}$ carbonate buffer at $\mathrm{pH} 9.5$ for $1 \mathrm{~h}$ at $37^{\circ} \mathrm{C}$. The plate was then refrigerated overnight at $4^{\circ} \mathrm{C}$. 
On the following day, the plate was thoroughly washed using 100 $\mu l$ of phosphate-buffered saline (PBS) and $0.05 \%$ Tween 20 . The wash was repeated twice using $100 \mu \mathrm{l}$ PBS and 5\% bovine serum albumin (BSA) for 15 min each. A $50-\mu$ l aliquot of PBS and $5 \%$ BSA was added to each well before adding the sample of $50 \mu \mathrm{l}$ of embryo supernatant. Amniotic fluid was used as a positive control. Amniotic fluid $(50 \mu \mathrm{l})$ and $50 \mu \mathrm{l}$ of pure blastocyst culture media (Irvine Scientific) in which no embryos had been cultured (the negative control) were incubated for a period of $1 \mathrm{~h}$ at $37^{\circ} \mathrm{C}$. After incubation, the plates were washed 3 times with PBS, followed by incubation with a specific biotin-conjugated $\mathrm{mAB}$ (w6/32 MCA81B; Serotec) at a 1:1,000 dilution in PBS and 1\% BSA for $45 \mathrm{~min}$ at $37^{\circ} \mathrm{C}$ and then washed 5 times with PBS. Streptavidin alkaline phosphatase conjugated (BD Bioscience Pharmingen, San Diego, Calif., USA) at a concentration of $1: 1,000$ in carbonate buffer was incubated for $30 \mathrm{~min}$ at $37^{\circ} \mathrm{C}$ and washed 5 times with PBS, after which phosphatase substrate was added at a concentration of $1 \mathrm{mg} / \mathrm{ml}$ in $10 \%$ diethanolamine at $\mathrm{pH} 9.8$ for 30 min. The colorimetric reaction was then stopped by the addition of $50 \mu \mathrm{l}$ of $3 \mathrm{~mol} / \mathrm{l} \mathrm{NaOH}$. The sHLA-G concentration was determined by absorbance at $405 \mathrm{~nm}$ on the EL800 Universal Microplate Reader (Bio-Tek Instruments Inc., Winooski, Vt., USA).

\section{Statistical Analysis}

Fisher's exact test was used to test for association between two categorical studied variables. The small marginal totals for some levels of the categories variables necessitate the use of this test. The agreement between the PB-I and PB-II and blastocyst was evaluated through the $\kappa$ statistic. The median levels of expression of sHLA-G for the categories of oocyte grade, PB-I, PB-II and blastocyst grade were calculated together with the interquartile range. To compare the median levels of sHLA-G between the levels of these variables, two statistical methods were used. Since oocyte grade has a strict ordinal structure, the estimation and testing of the Spearman correlation coefficient between sHLA-G and oocyte grade is an optimal test for the hypothesis of no difference in median sHLA-G levels across the categories of oocyte grade. For the remaining variables a Kruskal-Wallis test (nonparametric analysis of variance) was performed for the comparison of the median levels of soluble sHLA-G.

\section{Results}

Fifty-seven oocytes from donors ( $\leq 28$ years of age) were studied. The indication was that the majority of oocytes were aneuploid - CGH results for meiosis I of PB-I tested revealed that $21 / 57$ (36.8\%) were euploid, 33/57 (57.9\%) aneuploid and 3/57 (5.2\%) inconclusive (INC). Furthermore, CGH results for meiosis II of the PB-II tested revealed that 25/57 (44.0\%) were euploid, $27 / 57$ (47.4\%) were aneuploid, and 5/57 (9.0\%) were inconclusive (INC). All day-3 embryos underwent biopsy - removing a mononucleated blastomere. 45 of 57 embryos had $\geq 7$ blastomeres and were categorized grade 1 or 2 (78.9\%). CGH results for mitosis (day-3 mononucleated blastomeres) re- vealed that 23/57 (40.4\%) were normal, 31/57 (54.4\%) were abnormal, and 3/57 (5.3\%) were inconclusive (INC). The results were reported as inconclusive as a result of no cell, no amplification or no clear fluorescent staining/no interpretation.

Metaphase II oocytes were also graded, using light microscopy, the best quality was grade 1 and poorest quality was grade 3 (table 1). There were 33 aneuploid oocytes - 18/54 (54.5\%) originated from grade 1 (best quality) oocytes, the remaining 15 (45.5\%) originated from grade $2 / 3$ (poorer quality) oocytes. Furthermore, there were 21 euploid oocytes $-8 / 21$ originated from grade 1 (best quality) oocytes (38.1\%) and the remaining $13(61.9 \%)$ originated from grade $2 / 3$ (poorer quality) oocytes. No association was found between the best oocytes - grade 1 (highest quality) - and euploid chromosomal compliment of PB-I (Fisher's exact: $\mathrm{p}=0.758$ ). Furthermore, there was no association between oocyte grade and euploid chromosomal compliment of PB-II (the same as for PB-I). The majority of embryos were abnormal across all initial grades (Fisher's exact: $\mathrm{p}=0.548$ ).

There was no association between an oocyte's grade/ quality and subsequent blastomere biopsy's CGH results. The majority of blastomeres were aneuploid. $61 \%$ of the embryos that originated from the so-called 'best' quality oocytes were aneuploid and $58 \%$ of the embryos that originated from average/poor (grade $2 / 3$ ) oocytes were euploid as revealed by the CGH results from the blastomere biopsies (Fisher's exact: $\mathrm{p}=0.233$ ). However, our data showed there was a significant agreement between the CGH results of PB-I and PB-II, respectively, $\kappa=0.72$ $(\mathrm{SE}=0.11, \mathrm{p}<0.0001)$, indicating that PB-I and PB-II provided nearly the same information regarding their ploidy status. Furthermore, there was a significant agreement between PB-I and the blastomere biopsy CGH results. These two parameters also provide nearly the same information regarding their ploidy status, with $\kappa=0.84$ $(\mathrm{SE}=0.12, \mathrm{p}<0.0001)$. Interestingly, no association was observed between the CGH results of PB-I and subsequent blastocyst grade. The agreement between PB-II and the blastomere biopsy was similar to that of BP-I with $\kappa=0.75(\mathrm{SE}=0.11, \mathrm{p}<0.0001)$. There was no association between PB-II and subsequent blastocyst development. In both PB-II categories, euploid or aneuploid oocytes developed into about the same ratio of blastocysts. Interestingly, in cases where PB-I was euploid, 58\% reached the blastocyst stage, and where PB-I was aneuploid, $67 \%$ reached the blastocyst stage. Furthermore, in cases where blastomeres were euploid, $62 \%$ reached the blastocyst stage, and where blastomeres were aneuploid, 58\% 
Table 4. Data for all 57 oocytes

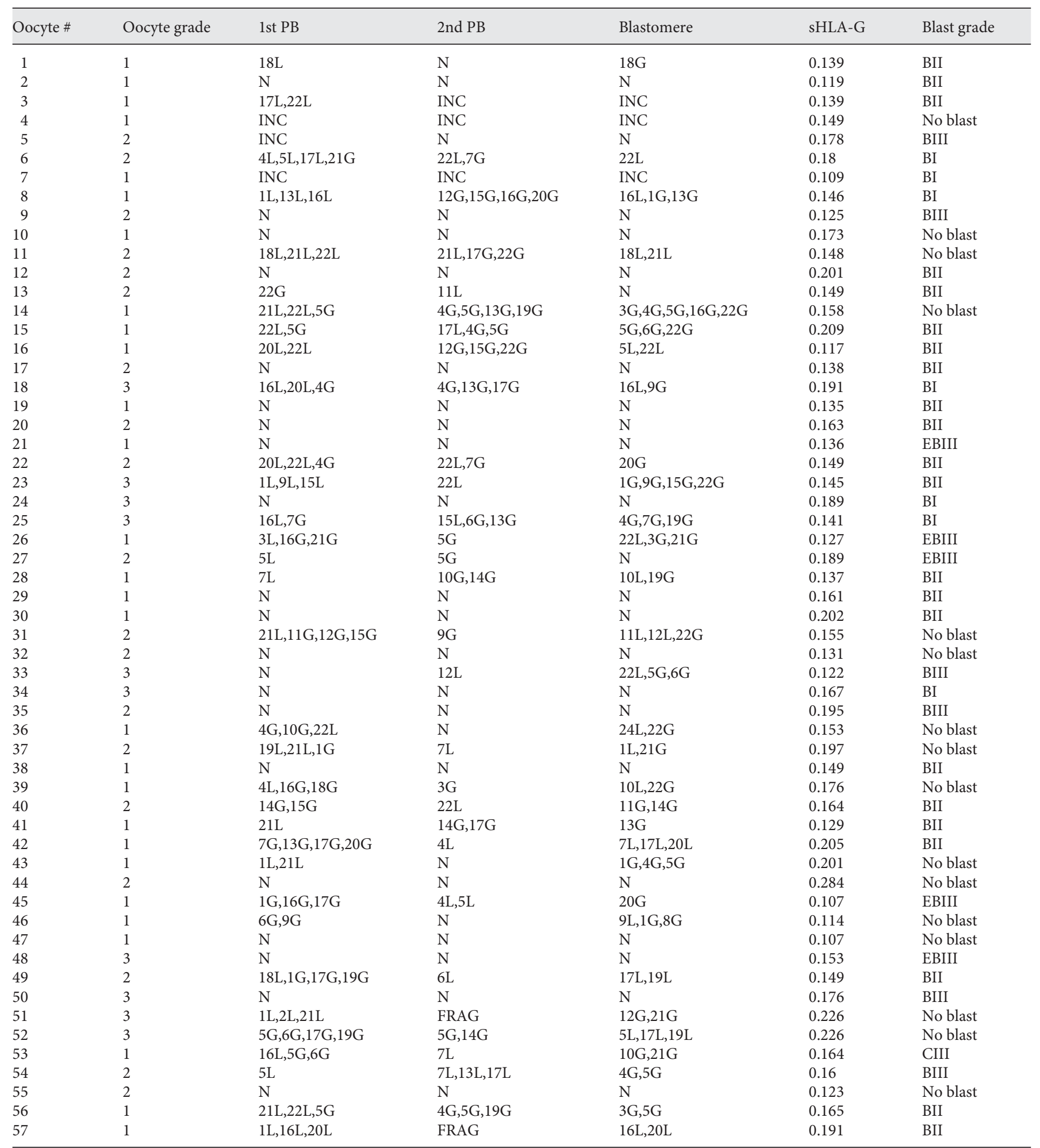

$\mathrm{L}=$ Loss; $\mathrm{G}=$ gain $\mathrm{N}=$ normal set of chromosomes; $\mathrm{FRAG}=$ polar body was fragmented and DNA amplification was not possible; 1 st $\mathrm{PB}=$ polar body $1 ; 2 \mathrm{nd} \mathrm{PB}=$ polar body 2 ; INC = DNA amplification was possible, but imaging and analysis was not possible. 
Table 5. CGH study pregnancy outcome

\begin{tabular}{ll}
\hline Embryo recipients & 5 \\
Age, years (average) & $37 \pm 1.5$ \\
Euploid blastocyst vitrified & 11 \\
Euploid blastocyst warmed & 11 \\
Euploid blastocyst that survived warming (\%) & $10 / 11(97.9)$ \\
Euploid blastocysts transferred (total) & 10 \\
Euploid blastocysts transferred (average) & 2.0 \\
Implantation/euploid blastocyst transferred (\%) & $5 / 10(50)$ \\
Live babies/euploid blastocyst transferred (\%) & $5 / 10(50)$ \\
Birth rate per warmed euploid blastocyst (\%) & $5 / 11(45.5)$ \\
Full-term pregnancies (\%) & $4 / 5(80)$ \\
Singletons & 3 \\
Twins (2 babies) & 1 \\
Triplets & 0 \\
Miscarriages & 0 \\
\hline
\end{tabular}

reached the blastocyst stage, indicating a lack of association (Fisher's exact: $p=0.923$ ). The findings were similar when the blastomere CGH results were correlated with blastocyst formation (Fisher's exact: $\mathrm{p}=0.770$ ).

There was no difference in the mean expression of sHLA-G between the categorical levels of PB-I, PB-II and blastocyst grade. However, oocyte grade and sHLA$\mathrm{G}$ expression was negatively correlated, with Spearman's correlation coefficient $-0.27(\mathrm{p}=0.0432)$. This is a weak correlation but reflects that the poorest oocyte grade had the highest expression (mean sHLA-G OD $=0.170 \pm$ 0.04) compared to best oocyte grade with lower expression (mean sHLA-G OD $=0.150 \pm 0.03$ ). The data for all 57 oocytes and their chromosomal status of PB-I, PB-II, day-3 blastomeres and sHLA-G respectively are presented in table 4. Implantation/pregnancy outcome of vitrified/warmed blastocyst were 50\% implantation, $80 \%$ pregnancy, three sets of singletons and one set of twins (table 5).

\section{Discussion}

Identifying euploid embryos prior to transfer in order to reduce the number of embryos for transfer, without sacrificing pregnancy rate and at the same time reducing high-order multiple gestations, have been of great interest to researchers. In our study we attempted to correlate the chromosomal status for PB-I, PB-II and blastomere, oocyte grading, embryo morphology score (GES) and sHLA-G (a biochemical embryo marker) in order to find any characteristic that could be used to assist in identify- ing/estimating of an embryo's viability and potential to develop into a live baby. We used 57 mature (MII) oocytes obtained from oocyte donors and fertilized with proven sperm donors to study their chromosomal status by CGH, and resulting 'normal' blastocysts were followed to determine if a live birth was obtained. The sequential chromosomal status of PB-I, PB-II and blastomere, respectively, was screened for by metaphase CGH. The rate of aneuploidy in PB-I and PB-II was 53\% in all oocytes and 54\% in subsequent day-3 embryos. A further breakdown indicated that the aneuploidy rates for PB-I (meiosis I) and PB-II (meiosis II) were 58 and 47\%, respectively. Any difference in ploidy between an oocyte's PB-I and PB-II could possibly be due to a rescue/correction mechanism, mosaicism, and malsegregation or cross-over errors during prophase I as described [22], or nondisjunction and premature separation as illustrated $[23,24]$ also speculated that missing signals might be due to the hybridization failure that can lead to misinterpretation.

Fragouli et al. [25] reported overall aneuploidy of 65\% in oocytes after CGH testing was performed. A further breakdown indicated that the aneuploidy rates for PB-I (meiosis I) and for PB-II (meiosis II) were 36 and 46\%, respectively. These results support the findings in our study regarding the genetic status of PB-I and PB-II. However, in another study by Fragouli et al. [26], they reported only $3 \%$ aneuploidy when young oocyte donors underwent PB-I CGH testing - these results contradict the findings in our study: we reported $58 \%$ of oocytes obtained from young egg donors were abnormal. Statistical analysis in the present study revealed that the correlation found between PB-I, PB-II and blastomere chromosomal results is good ( $\kappa>0.7$ for all three pairwise comparisons). Therefore, they could be used as a proxy for one another. However, we did find a negative association between embryo morphologic quality and corresponding genetic status of embryos on day 3. It was reported [27] that when embryo quality at the cleavage stage was correlated with chromosomal abnormalities, the morphological assessment might not reflect the chromosomal status of the embryo, because among the aneuploid embryos considerably high proportions were morphologically good quality, confirming our findings. We, therefore, suggest that an embryo's morphology cannot be correlated with its ploidy prior to transfer. This supports data by Munné et al. [28] suggesting that the best way to select euploid embryos for transfer is to use a combination of both morphologic and genetic testing. We reported that $31 / 50$ (62\%) of abnormal day-3 embryos developed into blastocysts. Our data indicated that there was no associa- 
tion between the expression of sHLA-G and embryo ploidy. In our study we reported a significant positive association between PB-I and blastomere ploidy which furthermore confirms that sperm derived from young healthy donors likely contributes very little to any additional aneuploidy after fertilization. Magli et al. [29] concluded that the major application of PB biopsy is the detection of maternally derived chromosomal aneuploidies and translocations in oocytes, and could be applied as a viable alternative to blastomere biopsy. In a large study by Li et al. [30], 42\% embryos were screened for 16, 18, 21 and $\mathrm{X}, \mathrm{Y}$ and underwent extended culture to the blastocyst stage and $20 \%$ progressed to the blastocyst stage. After retesting of the blastocyst's chromosomal complement, $61 \%$ were confirmed abnormal. This study confirms the similar high percentage of blastocyst aneuploidy as reported in our study.

Interestingly, Hassold and Jacobs [31] reported that at least $50 \%$ of first-trimester spontaneous miscarriages were chromosomally abnormal. Veiga et al. [32] reported that $26 \%$ of oocyte and zygote were aneuploid by FISH. Munné et al. [5] reported aneuploidy to be the most prominent chromosome anomaly found in normally developing monospermic embryos. Several other researchers (using FISH) have reported oocyte aneuploidy rates in excess of $50 \%$ in women older than 40 years of age $[8,10$, $11,33,34]$. Gutiérrez-Mateo et al. [35] reported 48\% aneuploidy after CGH was performed on PB-I. Montag et al. [36] concluded that the detection of the paternal influence on the genetic constitution of the embryo is not possible with $\mathrm{PB}$ diagnosis. However, $\mathrm{PB}$ diagnosis can be applied in cases of monogenetic diseases, as well as cases of maternal structural and numerical chromosome aberrations.

In all the above cases, only a limited number of chromosomes were evaluated (about one-quarter of the genome), leaving three-quarters untested. This leads to the assumption that several 'normal' embryos could have been aneuploid if the whole genome was screened. The conclusions drawn from these studies were based on incomplete genetic screening of embryos. The most recent study reported was a poster presentation at 2011 ESHRE, where Magli et al. [37] reported that 5 chromosomes (13, $16,18,21$, and $\mathrm{X}, \mathrm{Y}$ ) were analyzed and $50 \%$ were aneuploid. Furthermore, they reported that PB-I and PB-II do not reveal identical chromosomal information - which supports the findings in our study (table 4). Geraedts et al. [38] suggested that the PB approach be thoroughly investigated by a panel of researchers and reported annually to the ESHRE PGD Consortium [39]. Array CGH

screening of the first and second polar bodies added valuable information to the criteria needed in order to select a viable embryo for transfer as reported by Geraedts et al. [40] and Magli et al. [41]. In spite of the fact that more than half of mature (MII) oocytes obtained from young donors were aneuploid; the majority were fertilizable and subsequently developed into blastocysts. We hypothesize that the ability to fertilize eggs and subsequent extended embryo culture to blastocysts in no way affirms the chromosomal integrity of those blastocysts. The outcome of our small study and the transfer of vitrified/warmed blastocysts that originated from normal oocytes/zygotes and day-3 blastomeres as determined by sequential PGS of PB-I, PB-II and blastomere, respectively, resulted in encouraging implantation and pregnancy results and limiting high-order multiples (table 5). We conclude that future studies regarding sequential chromosomal screening of the whole genome to determine the chromosomal status of an embryo will shed more light on this controversial topic.

\section{Disclosure Statement}

This research paper is part of a $\mathrm{PhD}$ thesis at Stellenbosch University, South Africa, for candidate/researcher Dirk J. Kotze, MSc. Approval from the Committee of Human Research of the University of Stellenbosch was obtained (N06/07/119). All clinical research conducted was in full compliance with the guidelines as set forth by the American Society of Reproductive Medicine and adhered to ethical principles involving human subjects as defined by the Declaration of Helsinki in 1964.

References

Gynecol Obstet Invest 2012;74:304-312 870.
1 Alikani M, Cohen J, Tomkin G, Garrisi J, Mack C, Scott RT: Human embryo fragmentation in vitro and its implications for pregnancy and implantation. Fertil Steril 1999; 71:836-842.

-2 Schoolcraft WB, Surrey ES, Gardner DK: Embryo transfer: techniques and variables affecting success. Fertil Steril 2001;76:863-

- 3 Sher G, Fisch JD, Maassarani G, Matzner W: Antibodies to phosphatidylethanolamine and phosphatidylserine are associated with increased natural killer cell activity in nonmale factor infertility patients. Hum Reprod 2000;15:1932-1936.

4 Pehlivan T, Rubio C, Rodrigo L, Romero J, Remohi J, Simon C, Pellicer A: Impact of preimplantaion genetic diagnosis on IVF outcome in implantation failure patients. Reprod Biomed Online 2003;6:232-237. 
5 Munné S, Lee A, Rosenwaks Z, Grifo J, Cohen J: Diagnosis of major chromosome aneuploidies in human preimplantation embryos. Hum Reprod 1993;8:2185-2191.

6 Harton GL, Magli MC, Lundin K, Montag M, Lemmen J, Harper JC: European Society for Human Reproduction and Embryology (ESHRE) PGD Consortium/Embryology Special Interest Group. Hum Reprod 2011; 26:41-46.

7 Verlinsky Y, Ginsberg N, Lifchez A, Valle J, Moise J, Strom CM: Analysis of the first polar body: preconception genetic diagnosis. Hum Reprod 1990;5:826-828.

8 Kuliev A, Cieslak J, Ilkevitch Y, Verlinsky Y: Chromosomal abnormalities in a series of 6,733 human oocytes in preimplantation diagnosis for age-related aneuploidies. Reprod Biomed Online 2003;6:54-59.

-9 Wells D, Escudero T, Levy B, Hirschhorn K, Delhanty JD, Munné S: First clinical application of comparative genomic hybridization and polar body testing for preimplantation genetic diagnosis of aneuploidy. Fertil Steril 2002;78:543-549.

10 Gutiérrez-Mateo C, Wells D, Benet J, Sánchez-García JF, Bermúdez MG, Belil I, Egozcue J, Munné S, Navarro J: Reliability of comparative genomic hybridization to detect chromosome abnormalities in first polar bodies and metaphase II oocytes. Hum Reprod 2004;19:2118-2125.

-11 Fragouli E, Wells D, Thornhill A, Serhal P, Faed MJ, Harper JC, Delhanty JD: Comparative genomic hybridization analysis of human oocytes and polar bodies. Hum Reprod 2006;21:2319-2328.

12 Sher G, Keskintepe L, Keskintepe M, Ginsburg M, Maassarani G, Yakut T, Baltaci V, Kotze D, Unsal E: Oocyte karyotyping by comparative genomic hybridization provides a highly reliable method for selecting 'competent' embryos, markedly improving in vitro fertilization outcome: a multiphase study. Fertil Steril 2007;87:1033-1040.

-13 Sher G, Keskintepe L, Keskintepe M, Maassarani G, Tortoriello D, Brody S: Genetic analysis of human embryos by metaphase comparative genomic hybridization improves efficiency of IVF by increasing embryo implantation rate and reducing multiple pregnancies and spontaneous miscarriages. Fertil Steril 2009;92:1886-1894.

-14 Kallioniemi A, Kallioniemi OP, Sudar D, Rutovitz D, Gray JW, Waldman F, Pinkel D: Comparative genomic hybridization for molecular cytogeneic analysis of solid tumors. Science 1992;258:818-821.

15 Kirchhoff M, Rose Lundstee C: High-resolution comparative genomic hybridization in clinical cytogenetics. J Med Genet 2001;38: 740-744.

-16 Xia P: Intracyctoplasmic sperm injection: correlation of oocyte grade based on polar body, perivitelline space and cytoplasmic inclusions with fertilization rates and embryo quality. Hum Reprod 1997;12:1750-1755.
17 Fisch J, Rodriguez H, Ross R, Overby G, Sher G: The graduate embryo scoring system (GES) predicts blastocyst formation and pregnancy rates from cleaved-stage embryos. Hum Reprod 2001;16:1970-1975.

18 Dokras A, Sargent IL, Barlow DH: Human blastocyst grading: an indicator of developmental potential? Hum Reprod 1993;8:21192127.

19 Mukaida T, Nakamura S, Tomiyama T, Wada S, Oka C, Kasai M, Takahashi K: Vitrification of human blastocysts using cryoloops: clinical outcome of 223 cycles. Hum Reprod 2003; 18:384-391.

20 Mukaida T, Nakamura S, Tomiyama T, Wada S, Kasai M, Takahashi K: Successful birth after transfer of vitrified human blastocysts with use of a cryoloop containerless technique. Fertil Steril 2001;76:618-620.

21 Keskintepe L, Sher G, Keskintepe M: Reproductive oocyte/embryo genetic analysis: comparison between fluorescence in-situ hybridization and comparative genomic hybridization. Reprod Biomed Online 2007; 15: 303-309.

22 Schmerler S, Wessel GM: Polar bodies more a lack of understanding than a lack of respect. Mol Reprod Dev 2011;78:3-8.

23 Vialard F, Petit C, Bergere M, Gomes DM, Martel-Petit V, Lombroso R, Ville Y, Gerard $\mathrm{H}$, Selva J: Evidence of a high proportion of premature unbalanced separation of sister chromatids in the first polar bodies of women of advanced age. Hum Reprod 2006;21: 1172-1178.

24 Kuliev A, Zlatopolsky Z, Kirillova I, Spivakova J, Cieslak Janzen J: Meiosis errors in over 20,000 oocytes studied in the practice of preimplantation aneuploidy testing. Reprod Biomed Online 2011;22:2-8.

25 Fragouli E, Escalona A, Gutiérrez-Mateo C, Tormasi S, Alfarawati S, Sepulveda S, Noriega L, Garcia J, Wells D, Munné S: Comparative genomic hybridization of oocytes and first polar bodies from young donors. Reprod Biomed Online 2009;19:228-237.

26 Fragouli E, Katz-Jaffe M, Alfarawati S, Stevens J, Colls P, Goodall NN, Tormasi S, Gutierrez-Mateo C, Prates R, Schoolcraft WB, Munné S, Wells D: Comprehensive chromosome screening of polar bodies and blastocysts from couples experiencing repeated implantation failure. Fertil Steril 2010;94: 875-887.

27 Baltaci V, Satiroglu H, Kabukgu C, Onsal E, Aydinuraz B, Oner O, Aktas Y, Qetinkaya E, Turhan F, Aktan A: Relationship between embryo quality and aneuploidies. Reprod Biomed Online 2006;12;77-82.

28 Munné S, Chen S, Colls P, Garrisi J, Zheng X, Cekleniak N, et al: Maternal age, morphology, development and chromosome abnormalities in over 6,000 cleavage-stage embryos. Reprod Biomed Online 2007;14:628-634.
29 Magli MC, Gianaroli L, Ferraretti AP, Toschi M, Esposito F, Fasolino MC: The combination of polar body biopsy does not affect embryo viability. Hum Reprod 2004;19:11631169.

30 Li M, DeUgarte CM, Surrey M, Danzer H, DeCherney A, Hill DL: Fluorescence in situ hybridization reanalysis of day- 6 human blastocysts diagnosed with aneuploidy on day 3. Fertil Steril 2005;84:1395-400.

31 Hassold T, Jacobs P: Trisomy in man. Annu Rev Genet 1984;18:69-97.

32 Veiga A, Calderón G, Santaló J, Barri PN, Egozcue J: Chromosome studies in oocytes and zygotes from an IVF programme. Hum Reprod 1987;2:425.

33 Sandalinas M, Márquez C, Munné S: Spectral karyotyping of fresh, noninseminated oocytes. Mol Hum Reprod 2002;8:580-585.

- 34 Hassold T, Hall H, Hunt P: The origin of human aneuploidy: where we have been, where we are going. Hum Mol Genet 2007;16:203208

35 Gutiérrez-Mateo C, Benet J, Wells D, Colls P, Bermúdez MG, Sánchez-García JF, Egozcue J, Navarro J, Munné S: Aneuploidy study of human oocytes first polar body comparative genomic hybridization and metaphase II fluorescence in situ hybridization analysis. Hum Reprod 2004;19:2859-2868.

36 Montag M, van der Ven K, Rösing B, van der Ven H: Polar body biopsy: a viable alternative to preimplantation genetic diagnosis and screening. Reprod Biomed Online 2009; 18(suppl 1):6-11.

37 Magli MC, Grugnetti C, Castelletti E, Paviglianiti B, Gianaroli L: The study of aneuploidy on polar bodies predicts efficiently the chromosomal status of the corresponding oocyte. ESHRE 2011. Poster presentation (P413).

38 Geraedts J, Collins J, Gianaroli L, Goosens V, Handyside A, Harper J, Montang M, Repping S, Schmutzler A: What next for preimplantation genetic screening? A polar body approach. Hum Reprod 2010;25:575-577.

39 ESHRE PGD Consortium: Best practice guidelines for polar body and embryo biopsy for preimplantation genetic diagnosis/ screening (PGD/PGS). Hum Reprod 2011;26: 41-46.

-40 Geraedts J, Montag M, Magli MC, Repping S, Handyside A, Staessen C, Harper J, Schmutzler A, Collins J, Goossens V, van der Ven H, Vesela K, Gianaroli L: Polar body array CGH for prediction of the status of the corresponding oocyte. Part I: Clinical results. Hum Reprod 2011;26:3173-3180.

-41 Magli MC, Montag M, Köster M, Muzi L, Geraedts J, Collins J, Goossens V, Handyside AH, Harper J, Repping S, Schmutzler A, Vesela K, Gianaroli L: Polar body array CGH for prediction of the status of the corresponding oocyte. Part II: Technical aspects. Hum Reprod 2011;26:3181-3185. 